\title{
Adubação com fósforo em cana-soca e sua interação com magnésio
}

\author{
Fernando César Bachiega Zambrosi ( $\left.{ }^{*}\right)$ \\ Instituto Agronômico, Centro de Solos e Recursos Ambientais, Caixa Postal 28, 13012-970 Campinas (SP), Brasil. \\ $\left({ }^{*}\right)$ Autor correspondente: zambrosi@iac.sp.gov.br
}

Recebido: 6/fev./2012; Aceito: 17/jul./2012

\section{Resumo}

Existem dúvidas a respeito da eficiência da adubação fosfatada na cana-soca, bem como, em relação às interações do fósforo (P) com outros nutrientes que poderiam afetar as respostas da cultura à reaplicação de P. Realizou-se um estudo para avaliar os possíveis efeitos da aplicação de $\mathrm{P}\left(45\right.$ e $90 \mathrm{~kg} \mathrm{ha}^{-1}$ de $\mathrm{P}_{2} \mathrm{O}_{5}$ ) na ausência ou na presença de adubação com Mg (50 kg ha-1 de $\mathrm{Mg}$ ) sobre a produção de colmos da cana-soca. A adubação fosfatada promoveu acréscimos de 17\% na produção de colmos em relação ao tratamento controle (sem fertilizante fosfatado), porém, não dependeu da adubação com Mg, indicando que a aplicação deste nutriente não foi determinante para a ocorrência de resposta ao P. O estado nutricional em P da cana-de-açúcar também não foi influenciado pela aplicação de Mg, sendo alterado exclusivamente pela adubação fosfatada. O teor foliar de $P$ aumentou significativamente $(\mathrm{p}<0,05)$ de $1,4 \mathrm{~g} \mathrm{~kg}^{-1}$ no controle para $1,7 \mathrm{~g} \mathrm{~kg}^{-1}$ e $1,9 \mathrm{~g} \mathrm{~kg}^{-1}$ nos tratamentos que receberam 45 e $90 \mathrm{~kg} \mathrm{ha}^{-1}$ de $\mathrm{P}_{2} \mathrm{O}_{5}$ respectivamente. A eficiência de uso de fertilizante nitrogenado e potássico para a produção de colmos foi aumentada com a adubação fosfatada, com ganhos de $21 \%$ e $16 \%$ respectivamente. Os resultados sugerem que o incremento de produtividade na soqueira da cana-de-açúcar em solo com baixo teor disponível de P pode ser obtido com a reaplicação do nutriente, o que deve estar relacionado à melhoria do estado nutricional em $\mathrm{P}$ da cultura.

Palavras-chave: Saccharum, acúmulo de nutrientes, eficiência fertilizante, estado nutricional.

\section{Phosphorus fertilization in sugarcane ratoon and its interaction with magnesium}

\section{Abstract}

There are still doubts with regard to the efficiency of $\mathrm{P}$ fertilization in the sugarcane ratoon and the interaction between $\mathrm{P}$ and other nutrients that could affect the responses to $P$ reapplication. An experiment was set up to evaluate the effects of $P$ rates (45 and $90 \mathrm{~kg} \mathrm{ha}^{-1}$ of $\mathrm{P}_{2} \mathrm{O}_{5}$ ) applied either in the absence or presence of $\mathrm{Mg}$ fertilizer ( $50 \mathrm{~kg} \mathrm{ha}^{-1}$ of $\mathrm{Mg}$ ) on the stalk yield, of sugarcane ratoon. P fertilization increased stalk yield in 17\% compared to the control treatment (no P fertilizer). However, such response did not depend on $\mathrm{Mg}$ fertilization, suggesting that $\mathrm{Mg}$ was not critical to the occurrence of $\mathrm{P}$ response. The $\mathrm{P}$ nutritional status of sugarcane was not also affected by $\mathrm{Mg}$ application, being altered exclusively by $\mathrm{P}$ fertilization. P content in the leaves varied significantly $(p<0.05)$ from $1.4 \mathrm{~g} \mathrm{~kg}^{-1}$ in the control to $1.7 \mathrm{~g} \mathrm{~kg}^{-1}$ and $1.9 \mathrm{~g} \mathrm{~kg}^{-1}$ in the treatments with application of 45 and $90 \mathrm{~kg} \mathrm{ha}^{-1}$ of $\mathrm{P}_{2} \mathrm{O}_{5}$, respectively. P fertilization increased in $21 \%$ and $16 \%$, the efficiency in the use of $\mathrm{N}$ and $\mathrm{K}$ fertilizers, respectively, compared to the control. The results suggest that increases in the yield of sugarcane ratoon cultivated in a low-P soil can be obtained with $\mathrm{P}$ reapplication, what might be related to the improvements on the $\mathrm{P}$ nutritional status of the plants.

Key words: Saccharum, nutrients accumulation, fertilizer efficiency, nutritional status.

\section{INTRODUÇÃO}

A baixa disponibilidade de fósforo (P) no solo afeta de forma negativa o perfilhamento, desenvolvimento das folhas, comprimento e diâmetro dos entrenós da cana-de-açúcar, acarretando reduçôes de crescimento (Alvarez et al., 1960; Alvarez e Pacheco, 1963). Desta forma, a aplicação de $\mathrm{P}$ na cultura promove aumentos significativos na produção de colmos (Reis e Cabala-Rosand, 1986; Morelli et al., 1991). Porém, a mesma magnitude de resposta para a adubação com
$\mathrm{P}$ não é observada na cana-soca, com ganhos menos acentuados de produtividade em relaçáo à ausência da reaplicação de P (Korndorfer e AlCarde, 1992a). Esse padrão de resposta tem sido relacionado ao efeito residual do nutriente aplicado no plantio e à maior eficiência da cana-soca em acumular P (KorNdorfer e AlCARde, 1992b). Além disso, devido à adsorção do ânion fosfato na superfície dos minerais de argila e sua pequena mobilidade no solo (Fontes e WeED, 1996), considera-se que há baixo aproveitamento pelas soqueiras do nutriente recentemente aplicado. Estes fatores 
têm contribuído para recomendaçôes de menores quantidades de P (Prasad, 1976; Raij et al., 1997), ou mesmo, a ausência do nutriente nas formulações de fertilizantes comumente utilizadas para a cana-soca.

Embora seja argumentado que as doses de P utilizadas no plantio seriam suficientes para atender as exigências da cultura durante vários ciclos de crescimento, a disponibilidade do nutriente é significativamente diminuída ao longo do tempo (Korndorfer e AlCArde, 1992a; Korndorfer e Melo, 2009). Desta maneira, é possível que os níveis de $\mathrm{P}$ no solo não sejam compatíveis com a demanda da cana-de-açúcar, e também, para a manutenção do máximo potencial produtivo em cortes subsequentes. Esse processo pode ser exemplificado pelo estabelecimento de correlação positiva entre a produção de colmos no ciclo de segunda soqueira e o teor de P na camada subsuperficial (LANDell et al., 2003). Esse resultado reflete, portanto, o potencial de resposta da cana-soca aos incrementos na disponibilidade de $\mathrm{P}$ no solo, e a necessidade de avaliar os efeitos da reaplicação do nutriente.

Considerando que a resposta à adubação fosfatada é ainda influenciada pela interação com outros nutrientes, estudos envolvendo tal abordagem poderiam contribuir para avanços no manejo da adubaçáo para a cana-de-açúcar. Por exemplo, sugere-se importante interação entre $\mathrm{P}$ e $\mathrm{Mg}$, uma vez que este cátion participa da ativaçáo de enzimas quinases e da maioria das reaçóes que atuam na transferência de P (Marschner, 1995; Fageria, 2001). Esse fato deve estar relacionado aos efeitos negativos da condição de deficiência de $\mathrm{Mg}$ sobre a taxa de absorção e teores de P na raiz e parte aérea (FAgeria, 1983; Lasa et al., 2000), indicando assim, que a disponibilidade inadequada de $\mathrm{Mg}$ no solo poderia ser um fator limitante para respostas à adubação com P. Espera-se, portanto, que o estado nutricional em $\mathrm{Mg}$ da cana-soca seja favorecido por adubaçôes com este nutriente, e que possa resultar em ganhos de produtividade para a cultura.

De acordo com o exposto, o presente estudo foi desenvolvido com base na hipótese que o estado nutricional em $\mathrm{P}$ da cana-de-açúcar, e a resposta à adubação com esse nutriente na cana-soca são influenciados pela aplicação conjunta do fertilizante magnesiano. Desta maneira, objetivou-se estudar os possíveis efeitos da adubaçáo com $\mathrm{Pe}$ $\mathrm{Mg}$ sobre o estado nutricional, acúmulo de nutrientes nos colmos, e a produtividade da soqueira de cana-de-açúcar.

\section{MATERIAL E MÉTODOS}

O estudo foi realizado em condiçóes de campo, localizado em área produtora de cana-de-açúcar no município de Penápolis (SP), durante a safra agrícola 2010-2011. A área escolhida correspondia à cana de segunda soca da variedade SP81-3250, plantada em Latossolo VermelhoAmarelo, que vem sendo cultivado com cana-de-açúcar desde 2003. Em outubro de 2007, foram aplicados, respectivamente, 2,5 e $1,5 \mathrm{t} \mathrm{ha}^{-1}$ de calcário e gesso, seguindo-se a incorporação ao solo com arado de aiveca e grade intermediária. A área foi deixada em pousio até março de 2008, quando se realizou nova aração e gradagem antes do plantio da variedade SP81-3250. A adubação básica correspondeu a $40 \mathrm{~kg} \mathrm{ha}^{-1} \mathrm{de} \mathrm{N}, 180 \mathrm{~kg} \mathrm{ha}^{-1} \mathrm{de}_{2} \mathrm{O}_{5}$ e $150 \mathrm{~kg} \mathrm{ha}^{-1}$ de $\mathrm{K}_{2} \mathrm{O}$, com base em RaIj et al. (1997).

Após o primeiro corte em setembro de 2009, a adubação da soqueira consistiu de $110 \mathrm{~kg} \mathrm{ha}^{-1} \mathrm{de} \mathrm{N}$ e $150 \mathrm{~kg}$ $\mathrm{ha}^{-1}$ de $\mathrm{K}_{2} \mathrm{O}$. O experimento em cana-soca (segunda soqueira) foi instalado no início de novembro de 2010, aos 45 dias após o corte manual procedido de queima, em área uniforme quanto à brotação das plantas. Anteriormente ao início do experimento, o solo tinha as seguintes características químicas, respectivamente, para as camadas de $0-0,2 \mathrm{~m}$ e $0,2-0,4 \mathrm{~m}$ : matéria orgânica $=12$ e $10 \mathrm{~g} \mathrm{dm}^{-3} ; \mathrm{pH}$ em $\mathrm{CaCl}_{2}$ 0,01 $\mathrm{mol} \mathrm{L}^{-1}=5,1$ e 5,0 ; P-resina $=5$ e $3 \mathrm{mg} \mathrm{dm}^{-3}$; $\mathrm{K}=0,6$ e 0,$8 ; \mathrm{Ca}=14,0$ e 12,$0 ; \mathrm{Mg}=5,0$ e 4,$0 ; \mathrm{H}+\mathrm{Al}=23,0$ e 22,0; $\mathrm{Al}=1,0$ e $1,0 \mathrm{mmol}_{\mathrm{c}} \mathrm{dm}^{-3}$, determinadas segundo RaIj et al. (2001). O local foi escolhido pelo teor médio a baixo de Mg e muito baixo de P (RAIJ et al. 1997), bem como, pela disponibilidade de $\mathrm{Ca}$ e condiçóes de acidez não limitantes à cultura (RAIJ et al., 1997; Rossetto et al., 2004).

Os tratamentos corresponderam à combinação de $45 \mathrm{~kg} \mathrm{ha}^{-1}$ de $\mathrm{P}_{2} \mathrm{O}_{5}$ (P45) e $90 \mathrm{~kg} \mathrm{ha}^{-1}$ de $\mathrm{P}_{2} \mathrm{O}_{5}$ (P90), mais um tratamento-controle (sem aplicação de $\mathrm{P}, \mathrm{P} 0$ ), com a ausência de $\mathrm{Mg}(\mathrm{Mg} 0)$ ou adubação com $50 \mathrm{~kg} \mathrm{ha}^{-1}$ de $\mathrm{Mg}(\mathrm{Mg} 50)$, totalizando seis tratamentos. O delineamento experimental consistiu de blocos ao caso, com quatro repetições, sendo cada parcela formada por cinco linhas de cana-de-açúcar com dez metros de comprimento cada uma.

Anteriormente à aplicação dos fertilizantes, foram abertos sulcos em ambos os lados e distantes a aproximadamente $0,30 \mathrm{~m}$ da linha de plantio, a uma profundidade de $0,10-0,15 \mathrm{~m}$ com cultivador para cana-soca acoplado a um trator. Após a abertura dos sulcos, os fertilizantes fosfatado e magnesiano foram distribuídos manualmente em aplicação única, juntamente com $90 \mathrm{~kg} \mathrm{ha}^{-1}$ de $\mathrm{Ne} 130 \mathrm{~kg} \mathrm{ha}^{-1}$ de $\mathrm{K}_{2} \mathrm{O}$ (Raij et al., 1997). As fontes utilizadas foram o superfosfato triplo, nitrato de magnésio, nitrato de amônio e cloreto de potássio. Durante a realização do experimento, a precipitação pluvial total foi equivalente a $1187 \mathrm{~mm}$.

Para avaliação do estado nutricional da cana-de-açúcar, efetuou-se aos 3,5 meses da aplicação dos tratamentos, a amostragem da folha +1 (RAIJ et al., 1997). A coleta do experimento foi realizada após 11 meses do corte anterior (aos 9-10 meses da aplicação dos tratamentos). Em cada parcela experimental, a área útil correspondeu às três linhas centrais, desconsiderando-se dois metros iniciais em cada extremidade. Os colmos foram cortados manualmente, despalhados, separados dos ponteiros e pesados para quantificar a produtividade $\left(\mathrm{t} \mathrm{ha}^{-1}\right)$. Em cada parcela, subamostras de colmos foram coletadas, lavadas e secas em estufa de 
circulaçáo forçada de ar a $60^{\circ} \mathrm{C}$ para estimativa da umidade. A determinação dos teores de N, P, K, Ca, Mg e S nas amostras das folhas +1 e de colmos foi realizada segundo Bataglia et al. (1983). Com base nos resultados de massa seca e de teores, foram calculados os acúmulos $\left(\mathrm{kg} \mathrm{ha}^{-1}\right)$ de nutrientes nos colmos. A eficiência de uso de fertilizante nitrogenado (EUN) e potássico (EUK) em cana-soca foi calculada como segue: EUN ou EUK ( $\mathrm{t}$ colmos $\mathrm{kg}^{-1}$ de N ou $\mathrm{K}_{2} \mathrm{O}$ aplicado $)=\left(\right.$ produção de colmos, $\left.\mathrm{t} \mathrm{ha}^{-1}\right) /($ quantidade de $\mathrm{N}$ ou $\mathrm{K}_{2} \mathrm{O}$ aplicada, $\mathrm{kg} \mathrm{ha}^{-1}$ ).

Os resultados foram submetidos à análise de variância (ANOVA) e, posteriormente, realizadas as comparaçōes de médias pelos testes $\mathrm{F}$ e Duncan a $5 \%$ de probabilidade, com desdobramento dos fatores para o caso de interação significativa na ANOVA. Análises de correlação simples também foram estabelecidas entre variáveis previamente selecionadas.

\section{RESULTADOS E DISCUSSÃO}

Os resultados não confirmaram a hipótese do trabalho, de que a resposta da cana-soca à reaplicação de $\mathrm{P}$ seria influenciada pela adubaçáo com $\mathrm{Mg}$, uma vez que não foi observada interaçáo significativa $(p>0,05)$ entre os tratamentos de $\mathrm{P}$ e $\mathrm{Mg}$ para a produção de colmos. A aplicação de Mg também não afetou a produtividade da cana-soca, obtendo-se na média dos tratamentos de $\mathrm{P}$, o equivalente a 86,3 e 85,7 t ha $^{-1}$ de colmos, respectivamente, para $\mathrm{Mg} 0$ e $\mathrm{Mg} 50$. Existem, portanto, indicativos que o teor de $5 \mathrm{mmol}_{\mathrm{c}} \mathrm{dm}^{-3}$ de $\mathrm{Mg}$ na camada de $0-0,2 \mathrm{~m}$ náo seria limitante para a produção da cultura e ocorrência de respostas à adubação fosfatada. Esse resultado sugere, ainda, baixo potencial de resposta da cana-soca à adubação com $\mathrm{Mg}$, desde que, a quantidade aplicada de $50 \mathrm{~kg} \mathrm{ha}^{-1}$ seria suficiente para atender à demanda pelo nutriente (PRADO et al., 2002). A adubaçáo com 45 e $90 \mathrm{~kg} \mathrm{ha}^{-1}$ de $\mathrm{P}_{2} \mathrm{O}_{5}$, na média dos tratamentos de $\mathrm{Mg}$, aumentou em $17 \%$ a produção de colmos quando comparado ao $\mathrm{P} 0$, que náo recebeu fertilizante fosfatado (Figura 1). Entretanto, a aplicação de $45 \mathrm{~kg} \mathrm{ha}^{-1}$ de $\mathrm{P}_{2} \mathrm{O}_{5}$ foi provavelmente suficiente para atender à exigência por $\mathrm{P}$ da cana-soca, pois a produtividade para esta dose náo diferiu daquela obtida com $90 \mathrm{~kg} \mathrm{ha}^{-1}$ de $\mathrm{P}_{2} \mathrm{O}_{5}$. Os resultados deste trabalho, juntamente com aqueles obtidos por Korndorfer e
Alcarde (1992a), que observaram ganhos de produtividade após adubação fosfatada, demonstram o potencial da cana-soca para responder à reaplicação de $\mathrm{P}$ em solos com baixos teores do nutriente, reforçando a importância da adoção desta prática em áreas produtoras.

$\mathrm{O}$ estado nutricional em $\mathrm{P}$ da cana-de-açúcar não foi afetado pela adubação com $\mathrm{Mg}$, ou pela interação entre os tratamentos de $\mathrm{P}$ e $\mathrm{Mg}$ ( $\mathrm{p}>0,05)$. Porém, os teores de $\mathrm{P}$ nas folhas aumentaram em $21 \%$ e $36 \%$ em relação ao controle para P45 e P90 respectivamente (Tabela 1). Esses resultados corroboram KorNdORFER e ALCARDE (1992b), que verificaram efeito positivo da adubação fosfatada nos teores foliares de $\mathrm{P}$ em ciclo de cana-soca. Existem, portanto, indicativos do aproveitamento pela soqueira do fertilizante fosfatado recentemente aplicado. Ainda, esta melhoria do estado nutricional em $\mathrm{P}$ resultou em ganhos de produtividade da cultura, como sugerido pela correlação positiva entre os teores de $\mathrm{P}$ nas folhas e a produção de colmos $(r=0,44 ; \mathrm{p}=0,03 ; \mathrm{n}=24)$.

Além da não ocorrência de interação entre os tratamentos de $\mathrm{P}$ e Mg na ANOVA, a ausência de correlação significativa entre os teores foliares desses nutrientes $(r=0,17 ; p=0,43$; $\mathrm{n}=24)$ indicam que não houve relação entre eles. Com base no estado nutricional em $\mathrm{P}$ da cana-soca (Tabela 1), é plausível inferir que o teor de $5 \mathrm{mmol}_{\mathrm{c}} \mathrm{dm}^{-3}$ de $\mathrm{Mg}$ na camada superficial do solo não limitou a absorção e o transporte

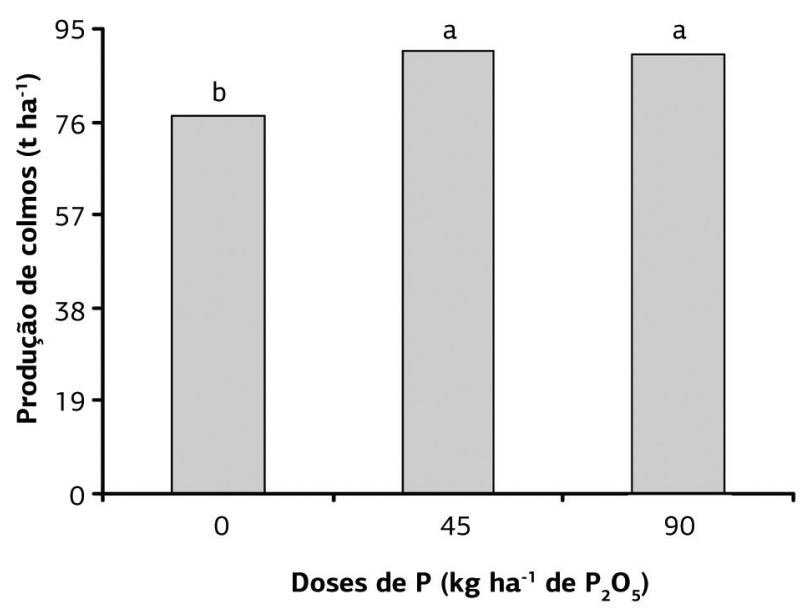

Figura 1. Produção de colmos no ciclo de cana-soca em função da adubaçáo com $\mathrm{P}$. As colunas com letras iguais não diferem pelo teste de Duncan a $5 \%$ de probabilidade.

Tabela 1. Teores de nutrientes $\left(\mathrm{g} \mathrm{kg}^{-1}\right)$ na folha diagnóstico aos 3,5 meses da adubação com $\mathrm{P}$ e $\mathrm{Mg}$ na cana-soca

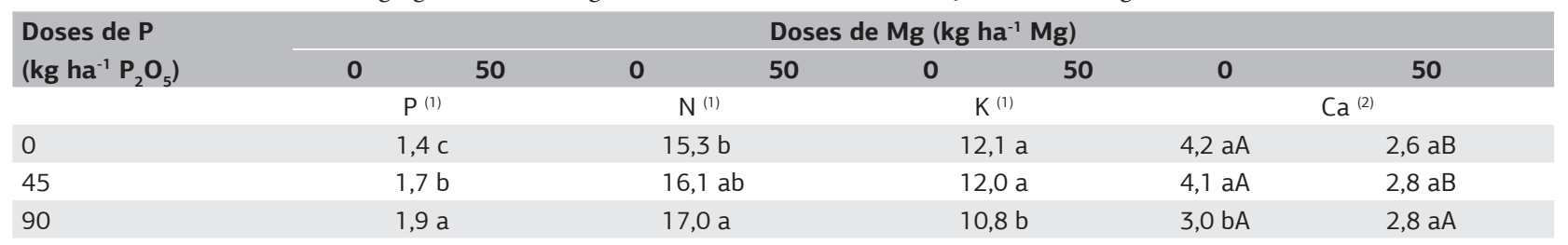

(1) Ausência de interaçáo significativa $(\mathrm{p}>0,05)$ entre os tratamentos de $\mathrm{P}$ e Mg. Os valores representam as médias das doses de Mg.

$\left.{ }^{2}\right)$ Interação significativa $(\mathrm{p}<0,05)$ entre os tratamentos de $\mathrm{P}$ e Mg.

As médias acompanhadas por letras minúsculas iguais na mesma coluna e por letras maiúsculas na mesma linha não diferem, respectivamente, pelos testes de Duncan e F a $5 \%$ de probabilidade. 
de P para a parte aérea e, assim, os efeitos da reaplicaçáo de $\mathrm{P}$ sobre os seus teores nas folhas. Desta maneira, é esperado que somente em condiçôes de deficiência severa por $\mathrm{Mg}$, como aquelas impostas em condiçôes controladas de solução nutritiva (FAGERIA, 1983; LASA et al., 2000), que o efeito deste cátion no estado nutricional em $\mathrm{P}$ das plantas seja manifestado em maior intensidade. De fato, em solo calcário, onde a concentração de sais na solução do solo é normalmente elevada, a aplicação de doses crescentes de $\mathrm{Mg}$ não resultou em estímulo à absorçáo do $\mathrm{P}$ adicionado via fertilizante (XIONg e ZHou, 1995). O teor foliar de $\mathrm{Mg}$, embora em pequena magnitude, aumentou significativamente $(\mathrm{p}<0,05)$ após aplicação do fertilizante contendo o nutriente. $\mathrm{Na}$ média dos tratamentos de $\mathrm{P}$, variou de 1,5 $\mathrm{g} \mathrm{kg}^{-1} \mathrm{em} \mathrm{Mg} 0$ para 1,8 $\mathrm{g} \mathrm{kg}^{-1} \mathrm{em} \mathrm{Mg} 50$, os quais estão na faixa de teor considerada adequada para a cana-de-açúcar de 1,0 a 3,0 $\mathrm{g} \mathrm{kg}^{-1}$, segundo RAIJ et al. (1997). Portanto, mesmo na ausência da adubação com $\mathrm{Mg}$, a análise foliar não indicou sua deficiência, o que deve, em parte, explicar a falta de resposta para a produção de colmos à adubação magnesiana.

Os teores foliares de $\mathrm{N}$ e $\mathrm{K}$ foram afetados exclusivamente pelas doses de $\mathrm{P}$, sem ocorrer interação significativa $(\mathrm{p}>0,05)$ com o $\mathrm{Mg}$, ou mesmo efeito da adubação com esse nutriente (Tabela 1). A dose mais elevada de $\mathrm{P}$ aumentou os teores de $\mathrm{N}$ em 11\% comparado ao controle. Além disso, foi observada correlação positiva entre $\mathrm{N}$ e $\mathrm{P}(\mathrm{r}=0,54 ; \mathrm{p}=0,01 ; \mathrm{n}=24)$, indicando aumentos concomitantes dos seus teores nas folhas, e a influência da disponibilidade de $\mathrm{P}$ sobre o estado nutricional em $\mathrm{N}$ da cana-soca. Esse fato poderia ser atribuído ao efeito do $\mathrm{P}$ sobre a absorçáo e o transporte de nitrato para a parte aérea (RufTy et al., 1990; JesChKe et al., 1997). Ao contrário do observado para o $\mathrm{N}$, os teores de $\mathrm{K}$ foram reduzidos com a aplicação de $90 \mathrm{~kg}$ $\mathrm{ha}^{-1}$ de $\mathrm{P}_{2} \mathrm{O}_{5}$ (Tabela 1). Efeito depressivo no teor de $\mathrm{K}$ em função da adubação fosfatada também foi reportado para a parte aérea da soja (Rosolem e Marcello, 1998). Em plantas de aveia que receberam até $80 \mathrm{~kg}$ $\mathrm{ha}^{-1}$ de $\mathrm{P}_{2} \mathrm{O}_{5}$, porém, os teores de $\mathrm{K}$ aumentaram nas folhas (NaKagawa e Rosolem, 2005), enquanto nenhum efeito de doses de P foi detectado para os teores de $\mathrm{K}$ em folhas de cebola (BoyHan et al., 2007). Esses resultados controversos não permitem estabelecer um padrão definido sobre o efeito da adubaçáo fosfatada no estado nutricional em $\mathrm{K}$ das plantas. É válido ressaltar, entretanto, que os teores foliares de $\mathrm{K}$ da cana-soca situaram-se na faixa adequada (10 a $16 \mathrm{~g} \mathrm{~kg}^{-1}$, RAIJ et al., 1997), sugerindo que a aplicaçáo de $\mathrm{P}$ associada a doses recomendadas de $\mathrm{K}$ não deve induzir deficiência de $\mathrm{K}$ e afetar negativamente a produtividade.

A ocorrência de interação significativa $(p<0,05)$ entre $\mathrm{P}$ e $\mathrm{Mg}$ foi detectada para os teores foliares de $\mathrm{Ca}$, demonstrando resposta diferencial para a adubação fosfatada em função da aplicação ou não de $\mathrm{Mg}$ (Tabela 1). Por exemplo, em $\mathrm{Mg} 0$, o P90 reduziu em $28 \%$ o teor de Ca comparado ao controle, enquanto esta resposta deixou de ocorrer em Mg50. Por sua vez, exceto em P90, a adubação com $\mathrm{Mg}$ reduziu os teores foliares de $\mathrm{Ca}$. O efeito negativo do $\mathrm{Mg}$ sobre a absorção de Ca é atribuído à competição entre estes cátions nos sítios de absorção na superfície radicular (FAGeria, 1973; Marschner, 1995), o que deve ter resultado na correlação negativa entre os seus teores nas folhas de cana-de-açúcar ( $r=-0,42 ; \mathrm{p}=0,03$; $\mathrm{n}=24)$. Entretanto, esta redução para o $\mathrm{Ca}$ não afetou negativamente a produção de colmos, desde que, os valores situaram-se na faixa de suficiência para a cultura (2,0 a 8,0 $\mathrm{g} \mathrm{kg}^{-1}$, RaIJ et al., 1997).

$\mathrm{O}$ acúmulo de nutrientes nos colmos da cana-soca foi influenciado pela adubação fosfatada, porém, não dependeu da aplicação de $\mathrm{Mg}$, como indicado pela ausência de interação significativa $(p>0,05)$ (Tabela 2$)$. Com exceção do $\mathrm{K}$, para a média dos tratamentos de $\mathrm{Mg}$, o acúmulo de nutrientes foi aumentado com a reaplicação de $\mathrm{P}$, porém sem diferenças entre P45 e P90. Por exemplo, o P45 aumentou em 2,6; 9,4; 5,1; 5,2 e 2,1 $\mathrm{kg} \mathrm{ha}^{-1}$ a quantidade acumulada de $\mathrm{P}, \mathrm{N}, \mathrm{Ca}, \mathrm{Mg}$ e $\mathrm{S}$, respectivamente, em relação ao P0 (Tabela 2). Esses resultados eram de certo modo esperados, desde que os aumentos foram relacionados aos ganhos de produtividade após a aplicação do $\mathrm{P}$ na soqueira (Figura 1), conforme suportado pelas correlaçóes positivas entre a produção de colmos e o acúmulo de $\mathrm{P}, \mathrm{Ca}$, $\mathrm{Mg}$ e $S$ nesta parte da planta $(\mathrm{r}=0,54$ a 0,$73 ; \mathrm{p}<0,0001$ a $<0,01 ; \mathrm{n}=24)$. Embora, a resposta à adubaçáo e aos teores foliares de $\mathrm{P}$ não tenha variado com a aplicação de $\mathrm{Mg}$, as quantidades acumuladas desses nutrientes nos colmos foram correlacionadas de forma positiva $(r=0,76 ; p<0,0001$; $\mathrm{n}=24$ ), indicando demanda mais elevada por $\mathrm{Mg}$ para sustentar maiores acúmulos de $\mathrm{P}$.

A eficiência de uso de fertilizante nitrogenado (EUN) e potássico (EUK) para a produçấo de colmos na cana-soca ( $\mathrm{t}$ de colmo $\mathrm{kg}^{-1}$ de $\mathrm{N}$ ou $\mathrm{K}_{2} \mathrm{O}$ aplicado) foi afetada exclusivamente pela fertilização com $\mathrm{P}$, sem ocorrer interação significativa $(\mathrm{p}>0,05)$ e efeito para $\mathrm{Mg}$ (Figura 2$)$.

Tabela 2. Acúmulo de nutrientes $\left(\mathrm{kg} \mathrm{ha}^{-1}\right)$ nos colmos da soqueira de cana-de-açúcar em função da adubaçáo com $P$

\begin{tabular}{|c|c|c|c|c|c|c|}
\hline $\begin{array}{l}\text { Doses de } P \\
\left(\mathrm{~kg} \mathrm{ha}^{-1} \mathrm{P}_{2} \mathrm{O}_{5}\right)\end{array}$ & $\mathbf{P}$ & $\mathbf{N}$ & K & $\mathrm{Ca}$ & Mg & $\mathbf{S}$ \\
\hline 0 & $5,7 b$ & $52,9 b$ & $28,0 \mathrm{a}$ & $23,0 \mathrm{~b}$ & $17,3 \mathrm{~b}$ & $3,8 \mathrm{~b}$ \\
\hline 45 & $8,3 \mathrm{a}$ & $62,3 \mathrm{a}$ & $34,8 \mathrm{a}$ & $28,1 \mathrm{a}$ & $22,5 \mathrm{a}$ & $5,9 \mathrm{a}$ \\
\hline 90 & $9,3 \mathrm{a}$ & $55,0 \mathrm{ab}$ & $30,1 \mathrm{a}$ & $31,5 \mathrm{a}$ & $24,4 \mathrm{a}$ & $5,4 \mathrm{a}$ \\
\hline
\end{tabular}

As médias acompanhadas por letras iguais na mesma coluna năo diferem pelo teste de Duncan a $5 \%$ de probabilidade. 


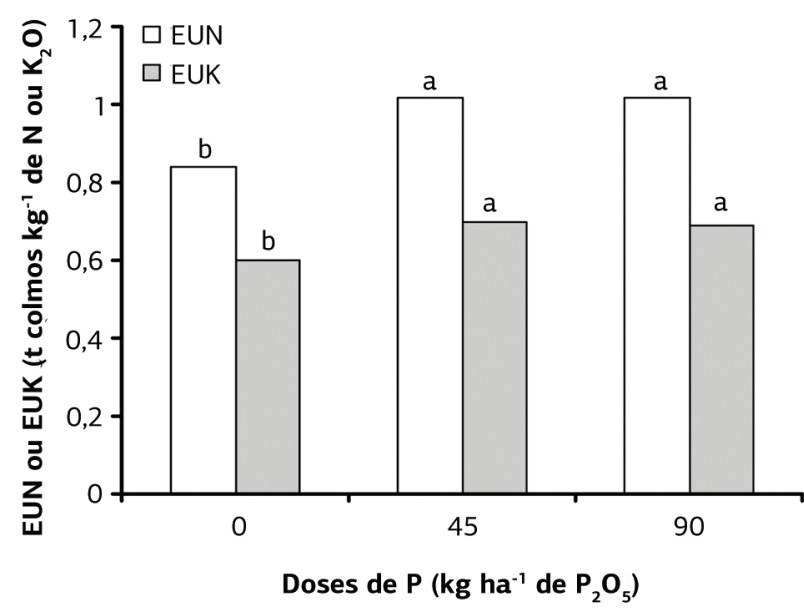

Figura 2. Eficiência de uso de fertilizante nitrogenado (EUN) e potássico (EUK) para a produção de colmos na soqueira da canade-açúcar em função da adubação com $\mathrm{P}$. As colunas com letras iguais para o mesmo parâmetro náo diferem pelo teste de Duncan a $5 \%$ de probabilidade.

A adubação fosfatada, de forma independente da dose, aumentou a EUN e EUK em $21 \%$ e $16 \%$ respectivamente, indicando maior produtividade para a mesma quantidade aplicada destes nutrientes. Isso resultou do estímulo à produção de colmos com a adoção da adubação fosfatada (Figura 1), o que reflete a importância desta prática para promover o aumento na eficiência de uso de $\mathrm{N}$ e K aplicados na cana-soca. De fato, este argumento é suportado por resultados conseguidos com outras culturas, para as quais, também foram observados efeitos positivos da adubação com P sobre a eficiência de uso de fertilizantes contendo N e K (WANG et al., 2010).

\section{CONCLUSÃO}

A adubaçáo fosfatada na soqueira da cana-de-açúcar melhora o estado nutricional da cultura em $\mathrm{P}$ e proporciona ganhos de produtividade em solo com baixo teor disponível do nutriente. Entretanto, em tais respostas não há relação com a aplicação conjunta de $\mathrm{Mg}$, devendo o teor equivalente a $5 \mathrm{mmol}_{\mathrm{c}} \mathrm{dm}^{-3}$ de $\mathrm{Mg}$ na camada superficial do solo $(0-0,2 \mathrm{~m})$ não ser limitante para a ocorrência de respostas à adubação fosfatada, ou mesmo para a produção de colmos em cana-soca.

\section{REFERÊNCIAS}

ALVAREZ, R.; AMARAL, A.Z.; ARRUDA, H.V. Ensaio de adubação N-P-K em cana-de-açúcar. Bragantia, v.19, p.1061-1069, 1960.

ALVAREZ, R.; PACHECO, J.A. Adubação da cana-de-açúcar: VII. Ensaio preliminar de adubação N-P-K no arenito Bauru. Bragantia, v.22, p.193-199, 1963.
BATAGLIA, O.C.; FURLANI, A.M.C.; TEIXEIRA, J.P.F; FURLANI, P.R.; GALLO, J.R. Método de análise química de plantas. Campinas: Instituto Agronômico, 1983. 48p. (Boletim técnico n.78)

BOYHAN, G.E.; TORRANCE, R.L.; HILL. C.R. Effects of nitrogen, phosphorus and potassium rates and fertilizer sources on yield and leaf nutrient status of short-day onions. HortScience, v. 42 , p.653-660, 2007.

FAGERIA, N.K. Absorption of magnesium and its influence on the uptake of phosphorus, potassium and calcium by intact groundnut plants. Plant and Soil, v.40, p.313-320, 1973.

FAGERIA, N.K. Ionic interactions in rice plants from dilute solutions. Plant and Soil, v.70, p.309-316, 1983.

FAGERIA, V.D. Nutrient interactions in crop plants. Journal of Plant Nutrition, v.24, p.1269-1290, 2001.

FONTES, M.P.F.; WEED S.B. Phosphate adsorption by clays from Brazilian Oxisols: relationships with specific surface area and mineralogy. Geoderma, v.72, p.37-51, 1996.

JESCHKE, W.D.; KIRKBY, E.A.; PEUKE, A.D.; PATE, J.S.; HARTUNG, W. Effects of P deficiency on assimilation and transport of nitrate and phosphate in intact plants of castor bean (Ricinus communis L.). Journal of Experimental Botany, v.48, p.75-91, 1997.

KORNDÖRFER, G.H.; ALCARDE, J.C. Aplicação de fósforo e rendimento de cana soca. Revista Brasileira de Ciência do Solo, v.16, p.183-186, 1992a.

KORNDÖRFER, G.H.; ALCARDE, J.C. Acúmulo e teor de fósforo em folhas de cana-de-açúcar. Revista Brasileira de Ciência do Solo, v.16, p.217-222, 1992b.

KORNDÖRFER, G.H.; MELO, S.P. Fontes de fósforo (fluída ou sólida) na produtividade agrícola e industrial da cana-de-açúcar. Ciência e Agrotecnologia, v.33, p.92-97, 2009.

LANDELL, M.G.A.; PRADO, H.; VASCONCELOS, A.C.M.; PERECIN, D.; ROSSETO, R.; BIDOIA, M.A.P.; SILVA, M.A.; XAVIER, M.A. Oxisol subsurface chemical attributes related to sugarcane productivity. Scientia Agricola, v.60, p.741-745, 2003.

LASA, B.; FRECHILLAL, S.; ALEU, M.; GONZÁLEZMORO, B.; LAMSFUS, C.; APARICIO-TEJO, P.M. Effects of low and high levels of magnesium on the response of sunflower plants grown with ammonium and nitrate. Plant and Soil, v.225, p.167-174, 2000.

MARSCHNER, H. Mineral Nutrition of Higher Plants. 2.ed. San Diego, CA, USA: Academic Press, 1995. 902p.

MORELLI, J.L.; NELLI, E.J.; BAPTISTELLA, J.R.; DEMATTÊ, J.L.I. Termofosfato na produtividade da cana-de-açúcar e nas propriedades químicas de um solo arenoso de baixa fertilidade. Revista Brasileira de Ciência do Solo, v.15, p.57-61, 1991.

NAKAGAWA, J.; ROSOLEM, C.A. Teores de nutrientes na folha e nos grãos de aveia-preta em função da adubação com fósforo e potássio. Bragantia, v.64, p.441-445, 2005. 
PRADO, R.M.; FERNANDES, F.M.; NATALE, W. Calcário e escória de siderurgia avaliados por análise foliar, acúmulo, e exportação de macronutrientes em cana-de-açúcar. Scientia Agricola, v.59, p.129-135, 2002.

PRASAD, M. Response of sugarcane to filter press mud and N, P, and $\mathrm{K}$ fertilizers. I. Effect of sugarcane yield and sucrose content. Agronomy Journal, v.68, p.539-543, 1976.

RAIJ, B. van; CATARELLA, H.; QUAGGIO, J. A.; FURLANI, A.M.C. Recomendaçôes de adubação e calagem para o Estado de São Paulo. 2.ed. Campinas: Instituto Agronômico, 1997. 285 p. (Boletim técnico, 100)

RAIJ, B. van; ANDRADE, J.C.; CANTARELLA, H.; QUAGGIO, J.A. Análise química para avaliação da fertilidade de solos tropicais. Campinas: Instituto Agronômico, 2001. 285p.

REIS, E.L.; CABALA-ROSAND, P. Respostas da cana-de-açúcar ao nitrogênio, fósforo e potássio em solo de tabuleiro do sul da Bahia. Revista Brasileira de Ciência do Solo, v.10, p.129-134, 1986.
ROSOLEM, C.A.; MARCELLO, C.S. Crescimento radicular e nutrição mineral da soja em função da calagem e adubaçâo fosfatada. Scientia Agricola, v.55, p.448-455, 1998.

ROSSETTO, R.; SPIRONELLO, A.; CANTARELLA, H.; QUAGGIO, J.A. Calagem para a cana-de-açúcar e sua interação com adubação potássica. Bragantia, v.63, p.105-119, 2004.

RUFTY Jr., T.W.; MACKOWN, C.T.; ISRAEL. D.W. Phosphorus stress effects on assimilation of nitrate. Plant Physiology, v.94, p.328-333, 1990 .

WANG, Y.; WANG, E.; WANG, D.; HUANG, S.; MA, Y.; SMITH, C.J.; WANG, L. Crop productivity and nutrient use efficiency as affected by long-term fertilization in North China Plain. Nutrient Cycling in Agroecosystems, v.86, p.105-119, 2010.

XIONG, L.M.; ZHOU, Z.G. Magnesium influence on plant uptake of phosphorus in a calcareous soil. Journal of Plant Nutrition, v.18, p.1251-1261, 1995. 